\title{
Minkowski Distance as a Quality of Service Assessment Tool
}

\author{
Yazeed Al-Sbou \\ Computer Science Department, Applied Science University, Kingdom of Bahrain.
}

ORCID: 0000-0001-9863-0606

\begin{abstract}
The aim of this paper is to describe an approach to assess the Quality of Service (QoS) of multimedia applications by considering their QoS parameters (i.e., delay, jitter, packet losses) requirements. Audio application was considered for this purpose because of the time-sensitive nature of their QoS requirements. In this work, the objective of QoS assessment is to evaluate the performance of the employed networks to investigate whether they satisfy the requirements of different applications that were sharing the same infrastructure over them. This involved devising a new QoS assessment technique that combines and summarizes the QoS parameters in a single value. This value represents the QoS level provided to the multimedia applications based on the network conditions compared to the QoS level expected for those applications. The devised assessment approach is based on Euclidean and Minkowski distance measures. The proposed approach simplifies the complexity of QoS assessment using simple and mathematically and very straightforward technique based on one equation and a simple mapping process. The obtained results using the devised approach reflects the actual QoS provided by the network for multimedia applications and were comparable to results obtained by some other proposed approaches.
\end{abstract}

Keywords: Quality of Service, Euclidean distance, Minkowski distance, multimedia applications, QoS Assessment.

\section{INTRODUCTION}

The use of networking systems is becoming a dominant factor in bringing information to users. As a result, the user requirements and attitudes have changed, demanding QoS levels other than the conventional Internet best-effort service. Implementing communication service levels that are higher than the best-effort level requires the measurement and assessment of network characteristics before any new transmission. Measurement techniques are traditionally used in telecommunications networks to support a wide range of activities including network planning and design, network operation and research [1]. Many real-time multimedia applications over the Internet have appeared today. These include audio, video phones, videoconferencing, video streaming, tele medical applications, distance learning, etc., with diverse requirements for their perceived quality. This gives rise to a need for assessing the quality of the transmitted applications in real time. The need to measure and assess the QoS is a fundamental requirement in modern communications systems for technical and commercial reasons. Currently, there is no standard for the QoS performance measurement; hence, various methods are used. These measurement and assessment techniques may be classified in different ways [2] [3] [4].

One type of classification is the distinction between direct and indirect measurements. Indirect measurement methods are based on network models and assumptions, where direct measurement methods do not rely on any models or expected behaviors but only on direct traffic observations at several points within the architecture. Another classification of measurement methods is by the distinction between real time and non-real time methods. Real time methods collect traffic data and packet events as they happen and some of them may be able to display the traffic information as it happens. In contrast, in non-real time measurement methods, the collected traffic data is analyzed offline (later) and may only be a subset (sample) of the total traffic population. Multimedia quality measurement may also be classified and carried out using two broad techniques: subjective and objective approaches. Generally, subjective tests of multimedia quality are based on evaluations made by human subjects under well-defined and controlled conditions therefore; the reference is the end user judgement which is directly captured using this approach. While, the objective methods measure the quality based on mathematical analysis that compare original and distorted multimedia signals.

A number of studies have used artificial intelligent techniques like fuzzy logic and neural networks for assessing network performance and QoS. For example, [5] [6] [7] [8] [9]. Fuzzy logic is a powerful tool that uses human reasoning as an important part of system design process. A major advantage of this feature is that it allows a natural description, in linguistic terms, of problems that should be solved rather than in terms of relationships rather than precise numerical values. Another advantage of the fuzzy system is that for some complex problems, it tends to be less computational intensive than other intelligent methodologies such as neural networks [5] [9].

In this paper, an alternative QoS assessment system was proposed relying on the principle of quantified distance evaluation between two vectors. This approach is based on the concept of Euclidean and Minkowski distance measures [10] [11] [12]. The distance system was proposed, as a nonintelligent system to be used as a baseline to be compared with the effectiveness of the fuzzy assessment system. Distance measure approach is usually used in multimedia processing as a similarity measure tool between two patterns that could be related to speech, image, graph, or signature [13] [14] [15] [16]. In this work, the use of distance approaches is justified by the absence of simple mathematical models or formulas to estimate the overall QoS. In addition, QoS assessment is a domain, which may meet the general conditions where the application of this approach may be considered appropriate. That is because 
QoS is a field where the value and ranges of the important QoS parameters can be represented numerically, i.e., the QoS parameters requirements of multimedia applications. Moreover, QoS assessment is a domain where the relationship between the input parameters and the output QoS exist but may be complicated. Distance approach simplifies this complexity by the proposed simple input-output relationship which is mathematically very straightforward and includes one equation and a simple mapping process.

\section{BASIC THEORY OF THE PROPOSED SYSTEM}

Similarity is a quantity that reflects the strength of relationship between two objects or two features. In other words, it is a numerical measure of how alike two data objects are. Distance measures the dissimilarity between two objects based on several features. These features can be represented as coordinate of the object in the features space. There are many types of distance calculation techniques that can be used to measure this dissimilarity. Dissimilarity is usually measured by Euclidean distance and Minkowski distance [10]. Euclidean distance or simply 'distance' evaluates the root of square differences between coordinates of a pair of objects.

$$
d_{i j}=\sqrt{\sum_{k=1}^{n}\left(x_{i k}-x_{j k}\right)^{2}}
$$

where $\mathrm{k}$ is the index of the object's coordinates, $\mathrm{x}_{\mathrm{i}}$ and $\mathrm{x}_{\mathrm{j}}$ are coordinates of the objects.

The Minkowski metric is widely used for measuring similarity between objects (e.g., images) [13]. Minkowski distance is the generalised distance as can be seen in (2) [17]. The Minkowski distance between two vectors may be defined as the geometric distance between two inputs with a variable scaling factor, power $(\lambda)$. When $\lambda$ is two it yields the Euclidian distance between two vectors. A disadvantage of the Minkowski method is that if one element in the vectors has a wider range than the other elements, the larger range may then 'dilute' the distances of the small-range elements.

$$
d_{i j}=\sqrt[\lambda]{\sum_{k=1}^{n}\left(x_{i k}-x_{j k}\right)^{\lambda}}
$$

\section{PROPOSED QOS ASSESSMENT DISTANCE APPROACH}

The use of non-intelligent method for measurements and evaluation of overall QoS is described here. A performance measurement method for estimating the actual network QoS experienced by the network users has been proposed based on distance measure approach. The results obtained using this approach were compared with those obtained using a fuzzy logic approach.

A general measurement system comprises four main processes: windowing, normalization, distance measurement and mapping. The functionality and role of each component will be briefly described as follows. The main QoS parameters for audio and videoconferencing applications are delay, jitter and packet losses. After measuring these parameters, they will be processed using a windowing technique, which means gathering every $m$ consecutive packets in one window (block) and calculating their average delay, jitter and packet loss. These parameters will be used as an input to the data transformation step. One weakness of the Minkowski distance function is that if an input element has relatively large values, then this value will dominate the other elements. Therefore, in this step, the distances were normalized by dividing the distance for each input attribute by specific numbers. These numbers represent the limits where the QoS will be poor. For videoconferencing, these limits were 600 msec for the delay, $30 \mathrm{msec}$ for the jitter, and 3\% for the packet loss. Similarly, for the audio, they were $600 \mathrm{msec}$ for the delay, $5 \mathrm{msec}$ for the jitter, and $6 \%$ for the loss. This was done in order to transform input data into a range which spans from 0 to 1 . In this case, all the elements under the root will have the same contribution in the evaluation process, which will prevent large values from dominating the distances of the small-range elements.

The mathematical procedure followed to compute the distance between the required and the measured QoS parameters is explained as follows. After transforming (i.e., normalizing) the input data (the required and the measured), the Minkowski distance are carried out as illustrated in (3) and (4). $X$ values represent the actual measurements (measured delay, measured jitter, and measured loss) and the $Y$ values represent required (desired) values (delay, jitter, and packet loss). The $Y$ values are application dependent.

$$
\begin{aligned}
& d_{X Y}=\sqrt[\lambda]{\sum_{i=1}^{n}\left(X_{i}-Y_{i}\right)^{\lambda}} \\
& \text { where } X_{i}=\left[D_{m}, J_{m}, L_{m}\right] \text { and } Y_{i}=\left[D_{r}, J_{r}, L_{r}\right] \\
& \text { Therefore; } d_{X Y}=\sqrt[\lambda]{\left(D_{m}-D_{r}\right)^{\lambda}+\left(J_{m}-J_{r}\right)^{\lambda}+\left(L_{m}-L_{r}\right)^{\lambda}} \\
& \qquad d_{X Y_{\text {nor }}}=\sqrt[\lambda]{\left(\frac{D_{m}-D_{r}}{600}\right)^{\lambda}+\left(\frac{J_{m}-J_{r}}{30}\right)^{\lambda}+\left(\frac{L_{m}-L_{r}}{3}\right)^{\lambda}}
\end{aligned}
$$

Where $d_{X Y}$ and $d_{X Y_{n o r}}$ are the regular and normalized distances respectively. $D_{m}, J_{m}$, and $L_{m}$ are the measured delays, jitter and loss, respectively. $D_{r}, J_{r}$, and $L_{r}$ are the required delays, jitter and loss, respectively.

The distance calculations of the measured values against the required values were carried out based on the Good QoS requirements (i.e., delay $\leq 150 \mathrm{msec}$, jitter $\leq 10 \mathrm{msec}$, and loss $\leq$ $1 \%)$. This means that the normalized QoS requirement are $\left\{D_{r}=\right.$ $150 \mathrm{msec}, J_{r}=10 \mathrm{msec}$, and $L_{r}=1 \%$ \}. Therefore, (3) becomes:

$$
d_{X Y_{\text {nor }}}=\sqrt[\lambda]{\left(\frac{D_{m}}{600}-0.25\right)^{\lambda}+\left(\frac{J_{m}}{10}-0.33\right)^{\lambda}+\left(L_{m}-0.33\right)^{\lambda}}
$$

As mentioned earlier, if $\lambda$ is selected to be equal to 2 , the equations correspond to the Euclidean Distance. The Euclidean distance (i.e., $\lambda=2$ ) has a problem if used in the evaluation system. From the equations above, it is obvious that the higher the distance ( $d_{X Y_{n o r}}$ ), the poorer the network during that transmission period. Initially, this method will provide a value 
for a network based on how far the measured QoS metrics deviated from the desired values regardless of the network actually performing better than desired. As an example of this is the case in which one or all the normalized measured values of the QoS metrics were less than the required values. The resulted Euclidean value would be a value, which reflects that the network has performed poorly but actually, the network has performed better than the desired requirement. The method presumes that the network has performed poorly because of the distance between the two values. That is because due to the square (i.e., $\lambda=2$ ) in the Euclidean distance formula, it does not take into account the sign between the parentheses. This also results in making the method unable to assess how "good" or how "poor" the network is performing. Therefore, $\lambda$ should be an integer odd number greater than one. In this case, the method will be able to presume the performance of the network. That is because if the output of the distance measurement system block in Figure 1 was less than zero, this implies that the network has performed better than the requirements, while if it was equal to zero, this means it has met the requirements. On the other hand, if the output was greater than zero, the network performed worse than desired.

In order to convert the output of the distance measurement step value to a quantity that reflect the QoS or to an indicator of how the network dealt with the application, a transformation of the output calculated distance is required to a value in the range $[0$, $100] \%$. This was carried out in the mapping step of the Figure 1. Suppose that $\lambda$ is selected to be 3 , the situation at which the distance $d_{X Y_{n o r}}$ is minimum is when the measured QoS metrics are zeros (i.e., $D_{m}=0 \mathrm{msec}, J_{m}=0 \mathrm{msec}$, and $L_{m}=0 \%$ ). Substituting this in (4), this produces a distance $d_{X Y_{\text {nor }}}=-0.444$. This case represents the best case of network performance (i.e., QoS $=100 \%)$. The worst network performance is when the measured metrics are equal or greater than the poor values, i.e. when $D_{m} \geq 600 \mathrm{msec}, J_{m} \geq 30 \mathrm{msec}$, and $L_{m} \geq 3 \%$. This gives $d_{X Y_{\text {nor }}}=1.01$ which corresponds to minimum poor QoS (i.e., QoS $=0$ ). Therefore, we have two pairs of $d_{X Y_{n o r}}$ and QoS as $(-0.444,100 \%)$ and $(1.01,0 \%)$. From this information, we can determine the equation of a straight line. Given that the line passes through the two points $\mathrm{P}_{1}=\left(x_{1}, y_{1}\right)$ (i.e., $(-0.444,100 \%)$ ) and $\mathrm{P}_{2}=\left(x_{2}, y_{2}\right)$ (i.e., $\left.(1.01,0 \%)\right)$, then the slope of the line is:

$$
m=\frac{y_{2}-y_{1}}{x_{2}-x_{1}}
$$

Given the slope $m$ and a point $\mathrm{P}_{1}=\left(x_{1}, y_{1}\right)$ through which the line passes, the relationship generally gets simplified algebraically to:

$$
y=m\left(x-x_{1}\right)+y_{1}
$$

If $y$ is replaced by QoS and $x$ is replaced by the $d_{X Y_{n o r}}$, the above equation can be rewritten as follows:

$$
Q o S=m * d_{X Y \text { nor }}+c
$$

where $c$ is constant equal to $\left(y_{1}-m x_{1}\right)$.

After calculating the slope $(m=-68.75),(6)$ becomes:

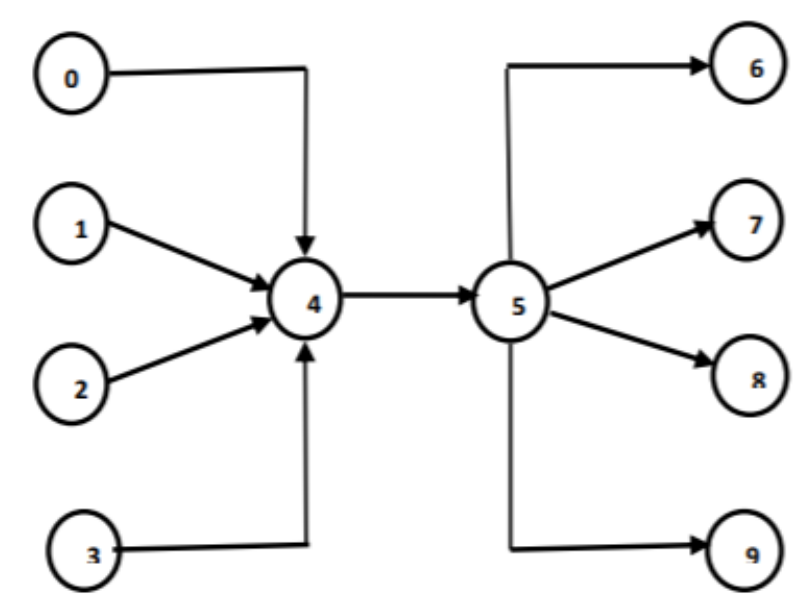

Fig. 1. Network topology

$$
Q o S=69.75-68.75 * d_{X Y \text { nor }}
$$

Similarly, when $\lambda$ is selected to be 5 and following the same previous steps, the final equation will be:

$$
Q o S=69.19-78.98 * d_{X Y} \text { nor }
$$

In order to generate the inputs used to test the proposed assessment system, different simulation scenarios were conducted using Network Simulator (NS) [18]. The network topology used for simulations was the same topology used in our previous study [7] and [19] as shown in Fig. 1. This network had four pairs of source/destination hosts. The pair between $\mathrm{N} 0$ and N6 was used for multimedia transmissions. Cross-traffic is also used to intercede between multimedia traffic and make the network busy during some selected times. The cross traffic pairs were between $\mathrm{N} 1$ and N7, N2 and N8

and N3 and N9. In addition, the following simulation specifications were used: UDP was the transport protocol; link capacity between every two nodes was $2 \mathrm{Mb} / \mathrm{sec}$, queue size of 50 packets.

\section{RESULTS AND DISCUSSIONS}

In this work, an assessment of audio multimedia application was carried out. Once the measured average QoS parameters (delay, jitter and loss) for the audio application were obtained, they were fed to the distance systems to produce the QoS. In order to test the accuracy of the proposed assessment system against the fuzzy logic one, combinations of samples of the input parameters were sampled and fed to both systems. These samples and their corresponding outputs are illustrated in Table 1.

From this Table, it can be seen that the output QoS values are a reflection of the input parameters based on the fuzzy rules used in the fuzzy system and the proposed procedure for the distance system. Moreover, the outputs of both systems are shown in Fig. 2. From Table 1 and Fig. 2, both assessment systems provided results, which are comparable to each other's. The discrepancies 
between the two systems were due to the different procedure followed by everyone. From the figures, distance system showed a higher variation and transitions than the fuzzy system. That was due to the fact that the fuzzy system is intelligent and governed by membership functions, Gaussian in our case, which may

Table 1. Sampled input QoS parameters and the assessed QoS using Distance and Fuzzy logic assessment approaches

\begin{tabular}{|c|c|c|c|c|c|}
\hline $\begin{array}{c}\text { Delay } \\
{[\mathrm{msec}]}\end{array}$ & $\begin{array}{c}\text { Jitter } \\
{[\mathrm{msec}]}\end{array}$ & $\begin{array}{c}\text { Loss } \\
{[\%]}\end{array}$ & $\begin{array}{c}\text { QoS using } \\
\text { distance } \\
\text { approach } \\
{[\%]}\end{array}$ & $\begin{array}{c}\text { QoS } \\
\text { using } \\
\text { Fuzzy } \\
\text { approach } \\
{[\%]}\end{array}$ & $\begin{array}{c}\text { QoS } \\
\text { Level }\end{array}$ \\
\hline 20 & 0.65 & 0.98 & 89.8 & 87.9 & Good \\
\hline 60 & 0.75 & 2.79 & 82.2 & 72.6 & Good \\
\hline 50 & 2.2 & 0.88 & 83.9 & 79.4 & Good \\
\hline 200 & 0.85 & 0.95 & 85.3 & 83.2 & Good \\
\hline 70 & 2.4 & 2.8 & 53.4 & 47.8 & Average \\
\hline 300 & 0.5 & 2.5 & 52.2 & 51.2 & Average \\
\hline 200 & 2.3 & 1.1 & 55.6 & 58.9 & Average \\
\hline 250 & 3.0 & 2.7 & 43.8 & 44.9 & Average \\
\hline 480 & 0.75 & 0.85 & 28.2 & 13.3 & Poor \\
\hline 75 & 4.0 & 0.98 & 28.1 & 18.3 & Poor \\
\hline 400 & 1.8 & 5.3 & 25.9 & 19.0 & Poor \\
\hline 550 & 4.3 & 5.5 & 10 & 9.73 & Poor \\
\hline
\end{tabular}

provide smooth transitions between the system states. On the other hand, the distance evaluation system is a non-intelligent approach, which mainly depends on the difference between the measured parameters values and the required thresholds and then combining (adding) the differences that will produce direct crisp values without any fuzzification.

The figures indicate that the fuzzy system provided QoS values in the range of [10\%-90\%] while the distance system generated QoS in the range of [0\%-100\%]. Therefore, the fuzzy system could not provide a QoS value less than $10 \%$ and a maximum value greater than $90 \%$. The cause of this effect was due to the overlaps between the input membership functions and between the output membership functions, which affected on the performance of the fuzzification and the defuzzification processes. On the contrary, the distance system, as mentioned before, relies on combining the differences between the measured and the desired values and therefore could produce an output range of [0-100] based on linear transformation.

To show the extent that the QoS was poor, average, and good, bar chart distribution was used. The length of the bar was representative of the percentage of each QoS case. Fig. 3 depicts the bar charts for the Audio application QoS using the fuzzy and the distance approaches, respectively. It can be observed that both assessment systems gave, relatively, similar results regarding the QoS level of the Audio application. It can be observed that this method provided a good picture about the measured QoS regions percentages.

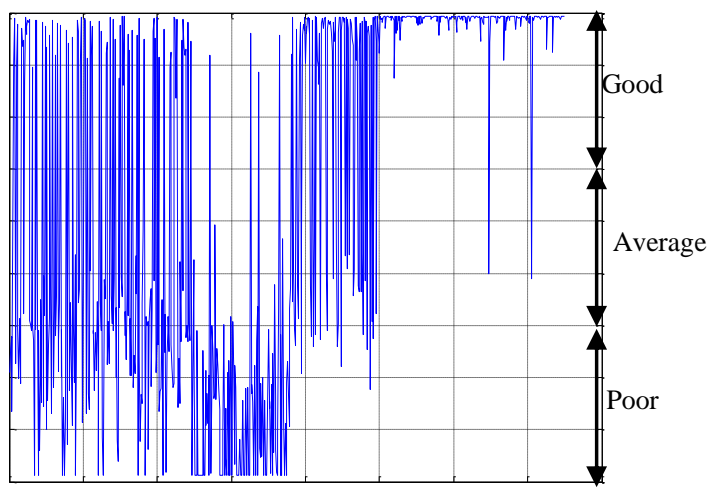

(a)

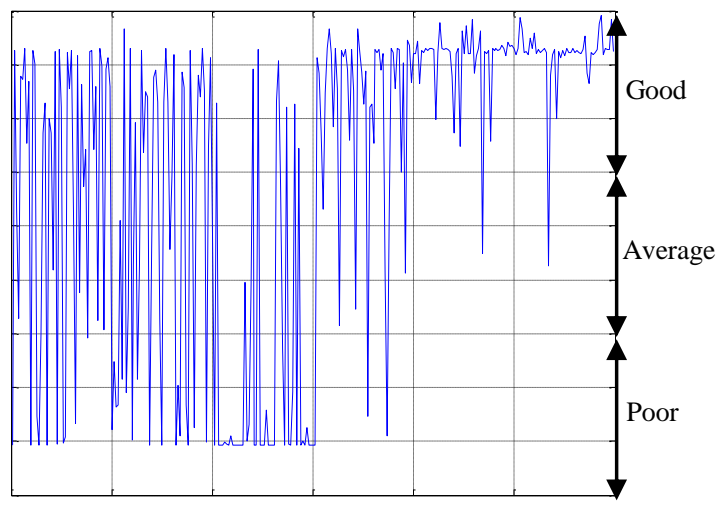

(b)

Fig. 2. The assessed QoS of Audio application using: (a) distance approach and (b) fuzzy logic approach.

Moreover, the distributions of the QoS were used in order to produce a more specific picture about the QoS of audio application QoS without classification of the QoS values into good, average and poor regions. The distributions are shown in Fig. 4. The figures illustrate the cumulative distributions, $\operatorname{Pr}\{X$ $<$ a\}, where the random variable $\mathrm{X}$ denotes the end-to-end QoS. The usefulness of this method stems from the fact that it gives the probability that the QoS is less than any threshold value in the 0 to 100 percentage range. For example, it can be seen from the figures that it is very easy to assess the probability or how many values of the QoS were less than $30 \%$ which are 0.24 and 0.2 using the fuzzy system and the distance system, respectively, which are comparable to each others. In addition, it can be observed that the minimum and maximum values of the QoS can be found from these figures. 


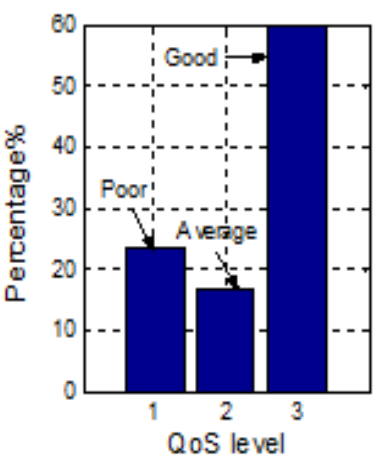

(a)

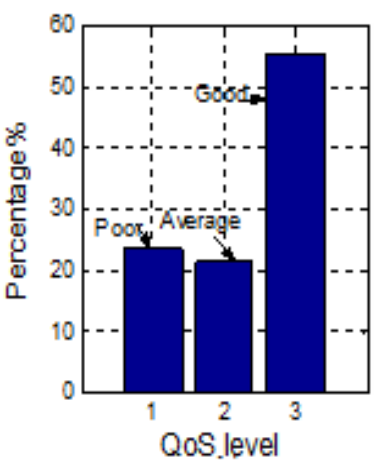

(b)
Fig. 3. The bar chart of Audio application QoS using: (a) fuzzy logic approach and (b) the distance approach.

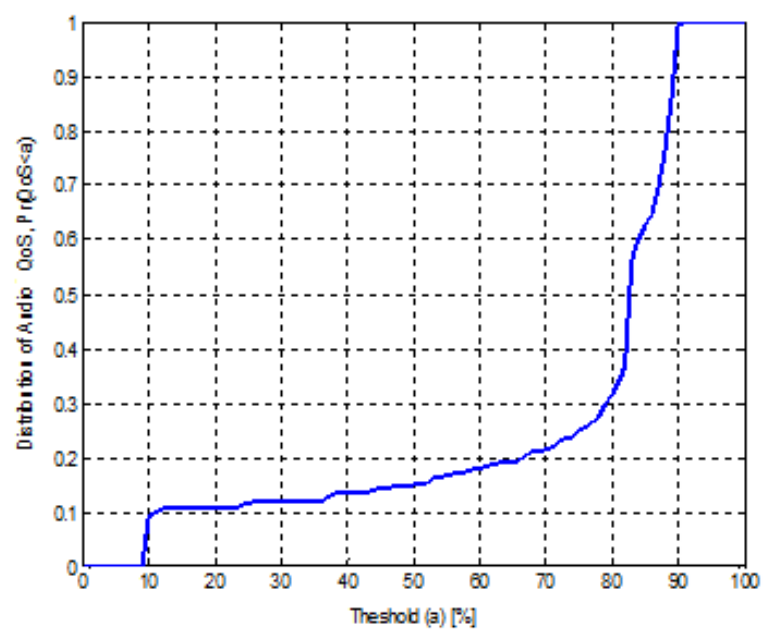

(a)

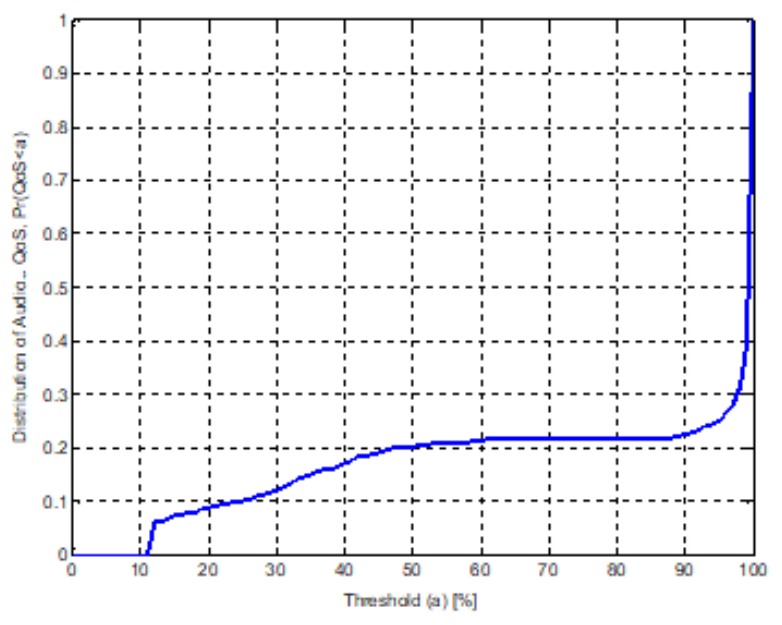

(b)

Fig. 4. Audio QoS distribution using: (a) fuzzy logic approach and (b) distancs approach.

\section{CONCLUSIONS}

This paper presented a new method to assess the QoS of multimedia applications: The Distance assessment system. The method showed how the QoS could be measured without the necessity for analytical models. The measured QoS has been classified into Good, Average, and Poor categories. In addition, for each application, based on the proposed system, the distributions and the overall QoS have also been obtained. The measured QoS using the proposed evaluation system was a good indication of the network conditions and resources availability.

\section{REFERENCES}

[1] ITU, "Quality of service regulation manual," https://www.itu.int/dms_pub/itu-d/opb/pref/D-PREFBB.QOS_REG01-2017-PDF-E.pdf, 2017.

[2] Salama A, Saatchi R and Burke D, "Quality of service evaluation and assessment methods in wireless networks," 4th International Conference on Information and Communication Technologies for Disaster Management (ICT-DM), Münster, 2017, pp. 1-6. doi: 10.1109/ICT-DM.2017.8275684).

[3] Pal D and Triyason T, "A Survey of Standardized Approaches towards the Quality of Experience Evaluation for Video Services: An ITU Perspective," International Journal of Digital Multimedia Broadcasting, 2018, pp. 1-25.

[4] Al-Sbou Y, "Quality of Service Monitoring of Wireless Networks Based on Combined Active-Passive Measurements," International Conference on Innovation, Technology, Enterprise and Entrepreneurship ICITEE 2019, Applied Science University, Kingdom of Bahrain, 2019, pp. 91-101.

[5] Al-Sbou Y, Salameh J, Al-Zoubi H, "Quality of Service Measurement Using Artificial Neural Networks,", International Review on Computers and Software, vol. 7, 2012, pp. 1584-1593.

[6] Saraireh M, Saatchi R, Shur U and Strachan R, "Fuzzy Logic Based Evaluation of Quality of Service for Multimedia Transmission," In Proceedings of PREP 2004. University of Hertfordshire, Hertfordshire, UK.

[7] Al-Sbou Y, "Fuzzy Logic Estimation System of Quality of Service for Multimedia Transmission,", International Journal of Quality of Service Issues in Networking (IJQoSIN), 2010, vol. 1.

[8] We T and Chen S, "A New Method for Constructing Membership Functions and Fuzzy Rules from Training Examples,: IEEE Transactions on Systems, Man and Cybernetics - Part B: Cybernetics, 29(1), 199, pp. 25-40.

[9] Oliveira R and Braum T, "A Fuzzy Logic Engine to Assist TCP Error Detection in Wireless Mobile Ad Hoc Networks," Next Generation Teletraffic and Wired/Wireless Advanced Networking New2an'04, 2004. 
[10] Teknomo K, "Euclidean Distance," http://people.revoledu.com/kardi/tutorial/Similarity/Euc lideanDistance.html, 2006. Last accessed on 1st February, 2020.

[11] . Merigo J M and Casanovas M, "A New Minkowski Distance Based on Induced Aggregation Operators," International Journal of Computational Intelligence Systems, vol. (2), 2011, pp. 123-133.

[12] Shirkhorshidi A S, Aghabozorgi S and Wah T, "A Comparison Study on Similarity and Dissimilarity Measures in Clustering Continuous Data," PLoS ONE, vol. 10, 2015.

[13] Li B, Chang E, and Wu C, "DPF - A Perceptual Distance Function for Image Retrieval," In Proceedings of IEEE International Conference on Image Processing, 2002, pp. 597-600.

[14] Eidenberger H, "Distance Measures for MPEG-7-based Retrieval," In Proceedings of ACM Multimedia Information Retrieval Workshop, ACM Multimedia Conference Proceedings, 2003, pp. 130 - 137.

[15] Wu S and Pols L, “A Distance Measure for Objective Quality Evaluation of Speech Communication Channels Using Also Dynamic Spectral Features," In Proceedings of the Institute of Phonetic Sciences Amsterdam, 1996, pp 27-42.

[16] Daoudi, K, "State of the Art in Speech and Audio Processing," http://www-rocq.inria.fr/imedia/Muscle /WP5/Docs/WP5_SoA_Task3_sept04.pdf.

[17] Batchelor B, "Pattern Recognition - Ideas in Practice," Plenum Press, 1978, New York, London.

[18] Bauer K and Sambhoos P, Network Simulator, University of Colorado at Boulder, http://www.isi.edu/nsnam/ns/.

[19] Al-Sbou Y,.Saatchi R,. Al-Khayatt S, Strachan R, Ayyash M, and. Saraireh M, "A novel quality of service assessment of multimedia traffic over wireless ad hoc networks," Proceedings of the Second IEEE International Conference on Next Generation Mobile Applications, Services, and Technologies, 2008, pp. 479-484. 